\title{
On the propagation, reflection, and transmission of transient cylindrical shear waves in nonhomogeneous four-parameter viscoelastic media
}

\section{T. Bryant Moodie}

The purpose of this paper is to study the propagation of cylindrical shear waves in nonhomogeneous four-parameter viscoelastic plates of arbitrary thickness. The plates have a transverse cylindrical hole and their material properties are functions of the radial distance from the center of this opening. They are initially unstressed and at rest. A suddenly rising shearing traction is applied uniformly over the boundary of the opening and parallel to the faces of the plates and thereafter steadily maintained; they are otherwise free from loading. We consider both the case of a finite plate with a stress-free cylindrical outer boundary, and an infinite plate composed of two media in welded contact along a cylindrical surface symmetrical with respect to the center of the opening. We find that a reflected pulse is produced at the outer boundary of the finite plate while reflected and transmitted pulses are produced at the interface in the infinite bi-viscoelastic plate. Ray techniques are used throughout, and formal asymptotic wavefront expansions of the solution functions are obtained. 


\section{Introduction}

Most of the problems involving linear viscoelastic taves that have been solved explicitly are for one-dimensional waves in homogeneous and isotropic media, for example, see the treatises by Bland [1] and Kolsky [11], and the references contained therein; also the references contained in the introduction of [5]. Recently some progress has been made in the treatment of multi-dimensional viscoelastic waves in homogeneous media, that is, see [2], [3], [5], [13], [15], [19], and the references contained therein: To partially overcome this lack of information concerning multidimensional waves in viscoelastic-media, and in particular for nonhomogeneous media, we present here a formal study of radially symmetric shear waves in nonhomogeneous four-parameter viscoelastic plates of arbitrary thickness. The four-parameter model chosen for our study exhibits an elastic, a viscous, and a retarded elastic response to shearing stress. Our viscoelastic material thus closely approximates the actual behaviour of a polymer [7].

The asymptotic methods to be employed in this paper originated in the field of optics. Luneburg [16] constructed a formal asymptotic expansion of the solution for the time-harmonic Maxwell equations, and demonstrated that the leading term of this expansion is the geometrical optics solution. Several authors, [6], [8], [9], [10], and [14], have shown that subsequent terms account for diffraction effects, that is, effects which cannot be accounted for by classical geometrical optics. Friedlander [6] formulated Luneburg's technique so that it applies to general progressing waves. It is Friedlander's formulation that we will employ in this paper.

Karal and Keller [8], using Friedlander's formulation to bypass the often difficult Fourier synthesis, extended Luneburg's technique to treat general progressing waves in nonhomogeneous isotropic elastic media. A partial justification, obtained by comparison with known solutions, has been given for the Karal-Keller technique of elastodynamics [4], [12], [17]. Cooper and Reiss [5] extended the Karal-Keller technique to study waves in linear homogeneous isotropic viscoelastic media, and Park and Reiss [18] then applied this extended Karal-Keller technique to study oscillatory impact of a standard nonhomogeneous viscoelastic rod. The latter authors included a partial justification of the method for 
homogeneous rods.

In this paper we extend the work of Glauz and Lee [7] and consider cylindrical pulse waves propagating in nonhomogeneous four-parameter viscoelastic plates of arbitrary thickness. To use the inherent symmetry of our problem and thereby simplify the analysis, we introduce the circular cylindrical coordinates $(r, \theta, z)$ and let the plates under consideration occupy $r_{0} \leq r \leq r_{1},|z| \leq d / 2$, and $r_{0} \leq r<\infty,|z| \leq d / 2$, for finite and infinite plates, respectively, where $r_{0}$ is the radius of the cylindrical opening in both plates, $r_{1}$ is the outer radius of the finite plate, and, in both cases, $d$ is the arbitrary plate thickness. For both plates the transverse cylindrical openings are subjected to shearing tractions which are uniformly distributed over the boundaries of these openings and act parallel to the faces of the plates. These tractions are suddenly applied and thereafter steadily maintained. The finite plate has a stress-free boundary at $r_{1}\left(r_{1}>r_{0}\right)$ and the infinite plate has an interface at $\bar{r} \quad\left(r_{0}<\bar{r}<\infty\right)$ across which the properties of the medium may be discontinuous. The only non-vanishing components of stress, strain, velocity, and displacement are $\boldsymbol{s}_{r \theta} \equiv s(r, t), \varepsilon_{r \theta} \equiv \varepsilon(r, t)$, $v_{\theta} \equiv v(r, t)$, and $u_{\theta} \equiv u(r, t)$, respectively, where $v \equiv \partial u / \partial t$.

Because proofs are not provided, the methods presented in this paper are formal. The expansions obtained are not necessarily convergent but presumably are asymptotic to the solutions of the equations of motion for our viscoelastic media. It is hoped that the results of this paper will shed some light on the way in which a cylindrical pulse propagates through a polymer and is reflected at a free surface or reflected and trensmitted at an interface between two media. To our knowledge this is the first time that any attempt has been made to solve the problems posed in this paper.

\section{Formulation}

We formulate the problems to be discussed within the context of the linear theory of viscoelasticity [1]. In a linear isotropic viscoelastic material, each shear (deviatoric) component of strain is related by the stress-strain law solely to the corresponding shear component of stress, 
and the relationship is the same for each component. We choose as a model on which to base the behaviour of our viscoelastic material, a fourparameter viscoelastic element which, when subjected to shearing stress, exhibits an elastic, a viscous, and a retarded elastic response. This four-parameter element must then consist of a two-parameter Maxwell element in series with a two-parameter Voigt-Kelvin element [1]. The stress-strain law for our four-parameter element, which exhibits the desired behaviour under shearing stress, is

(1)

$$
\begin{aligned}
\varepsilon & =\varepsilon_{1}+\varepsilon_{2}+\varepsilon_{3}, \\
\mu_{1} \varepsilon_{1} & =s, \\
\mu_{2} \varepsilon_{2}+\eta_{2} \varepsilon_{2, t} & =s, \\
\eta_{3} \varepsilon_{3, t} & =s,
\end{aligned}
$$

where $\varepsilon$ is the overall shear strain of the four-parameter element, $s$ the corresponding shear stress; $\varepsilon_{1}, \varepsilon_{2}$, and $\varepsilon_{3}$ are the shear strains associated with the Maxwell spring, the two-parameter Voigt-Kelvin element, and the Maxwell dashpot, respectively; $\mu_{1}$ and $\mu_{2}$ are the moduli of rigidity associated with the Maxwell and Voigt-Kelvin elements, respectively, and $n_{2}$ and $n_{3}$ are the moduli of shear viscosity associated with these elements. Assuming that the properties of the smallest portions into which we can conceive our viscoelastic material to be divided are the same as those of the substance in bulk, and eliminating $\varepsilon_{1}, \varepsilon_{2}$, and $\varepsilon_{3}$ from equations (I), we obtain the stress-strain relation for our viscoelastic solid to be

(2) $s_{t t}+\left[\left(\mu_{1} / n_{3}\right)+\left(\mu_{1} / \eta_{2}\right)+\left(\mu_{2} / \eta_{2}\right)\right] s_{t}+\left(\mu_{1} \mu_{2} / \eta_{2} n_{3}\right) s$ $=\mu_{1} \varepsilon_{t t}+\left(\mu_{1} \mu_{2} / n_{2}\right) \varepsilon_{t}$.

In equation (2), $s$ is the non-vanishing component of shear stress introduced in the preceding section, and $\varepsilon$ is the corresponding component of strain. The subscript $t$ appearing in equations (1) and (2) represents partial differentiation with respect to time $t$. The moduli of rigidity and viscosity are functions of the radial distance $r$ from the center of the cylindrical opening and are independent of $t$. 
The non-vanishing component of shear strain $\varepsilon$ can be expressed in terms of the single non-vanishing component of displacement $u$ by the relation [17],

$$
\varepsilon=\partial u / \partial r-u / r .
$$

Newton's second law gives the equation of motion for the viscoelastic plates as (neglecting body forces)

$$
\partial s / \partial r+2 s / r=\rho u_{t t},
$$

where $\rho=\rho(r)$ is the variable mass density of the medium. Using (3) in (2), and then eliminating $u$ between the resulting expression and equation (4), we obtain the partial differential equation for $s(r, t)$, that is, (5) $s_{t t t}+\left[\left(\mu_{1} / \eta_{3}\right)+\left(\mu_{1} / n_{2}\right)+\left(\mu_{2} / n_{2}\right)\right] s_{t t}+\left(\mu_{1} \mu_{2} / n_{2} n_{3}\right) s_{t}$

$$
=r \mu_{1}\left[(\partial / \partial t)+\left(\mu_{2} / n_{2}\right)\right]\left\{(\partial / \partial r)\left[\left(s_{r}+2 s / r\right) / r \rho\right]\right\} .
$$

We now assume [6] that the solution $s(r, t)$ of equation (5) may be represented by the series

$$
s \sim \cdot \sum_{n=0}^{\infty} A_{n}(r) F_{n}[t-S(r)], A_{0} \neq 0,
$$

where the $F_{n}$ 's are related by

$$
F_{n}^{\prime}=F_{n-1}, \quad n=1,2, \cdots,
$$

and it is assumed that $A_{n} \equiv 0$ for $n<0$. We further assume that the derivatives of $s$ may be obtained by term-wise differentiation in (6). The prime in (7) denotes differentiation with respect to the entire argument $(t-S)$, and (7) enables us to relate all of the $F_{n}$ 's to $F_{0}$ (the waveform) by successive integrations. For example, if $F_{0}$ is the Heaviside unit function $B(t)$ defined by

$$
H(t)=1 \text { for } t>0, \quad H(t)=0 \text { for } t<0 \text {; }
$$

then, by successive integrations, we obtain

$$
F_{n}=(t-S)^{n} H(t-S) / n !
$$

Note that $F_{0}$ vanishes for negative argument, that is, in front of the 
wavefront whose equation is given by $t=S(r)$, where $S$ is called the phase or eikonal function. For $F_{n}$ given by (9), the coefficients $A_{n}$ in (6) are the jump conditions for the stress and its derivatives across the wavefront. Solutions of (5) in the form (6) offer a convenient device for studying the propagation of stress discontinuities.

\section{Solution}

By seeking the solution of equation (5) in the form (6) [8], we find that the phase function $S$ satisfies the eikonal equation of geometrical optics,

$$
\left(S^{\prime}\right)^{2}=\rho / \mu_{1}=1 / c^{2},
$$

where $c=c(r)$ is the variable wave velocity for elastic shear waves in a medium whose rigidity is $\mu_{1}$. The amplitude functions $A_{n}$ satisfy the transport equations

$$
\begin{gathered}
2 S^{\prime} A_{n}^{\prime}+\rho\left(1 / \eta_{2}+1 / \eta_{3}\right) A_{n}+S^{\prime}\left(1 / r-\rho^{\prime} / \rho\right) A_{n}+S^{\prime \prime} A_{n}=P_{n}, n=0,1, \ldots, \\
P_{n} \equiv A_{n-1}^{\prime \prime}+\left[\left(\frac{1}{r}-\frac{\rho^{\prime}}{\rho}\right)-2 \lambda S^{\prime}\right] A_{n-1}^{\prime}-\left[\frac{\rho \lambda}{n_{3}}+\frac{2}{r}\left(\frac{2}{r}+\frac{\rho^{\prime}}{\rho}\right)\right. \\
\left.+\lambda S^{\prime \prime}+\lambda\left(\frac{1}{r}-\frac{\rho^{\prime}}{\rho}\right) S^{\prime}\right] A_{n-1}+\lambda A_{n-2}^{\prime \prime}+\lambda\left(\frac{1}{r}-\frac{\rho^{\prime}}{\rho}\right) A_{n-2}^{\prime}-\frac{2 \lambda}{r}\left(\frac{2}{r}+\frac{\rho^{\prime}}{\rho}\right) A_{n-2}, \\
\lambda(r) \equiv \mu_{2} / \eta_{2} .
\end{gathered}
$$

The eikonal equation (10) tells us that the stress discontinuities propagate through the viscoelastic medium with the velocity $c(r)$. The rays, which must cut the surfaces $S=$ const. at right angles, are straight lines. Integrating the eikonal equation along a ray we obtain

$$
S(r)=S\left(r_{0}\right) \pm \int_{r_{0}}^{r} d \tau / c(\tau),
$$

where the plus sign is associated with waves travelling in the direction of increasing $r$, that is, with outgoing waves, and the minus sign is associated with waves travelling in the direction of decreasing $r$, that is, with incoming waves. Equation (12) enables us to determine the phase at any point on a ray in terms of its value at $r=r_{0}$. It is very important when using our asymptotic method to choose the proper sign in 
equation (12) so that the direction of propagation is taken into account.

Our method is essentially one which reduces partial differential equations to ordinary differential equations. Often the latter can be solved to yield explicitly the desired asymptotic expansions. In some cases, however, the ordinary differential equations cannot be solved explicitly. This is a limitation of the method which is often overlooked [14].

The general solution of the transport equations (11) is

$$
\begin{gathered}
A_{n}(r)=A_{n}\left(r_{0}\right)\left[p(r) / p\left(r_{0}\right)\right]^{\frac{1}{2}} \exp \left\{\mp \int_{r_{0}}^{r} \gamma(\alpha) d \alpha\right\} \\
\pm\left(\frac{z}{2}\right) \int_{r_{0}}^{r} c(\tau)[p(r) / p(\tau)]^{\frac{2}{2}} \exp \left\{ \pm \int_{r}^{r} \gamma(\alpha) d \alpha\right\} P_{n}^{ \pm}(\tau) d \tau, n=0,1, \ldots, \\
p(r) \equiv \rho c / r, \gamma(r) \equiv \frac{\rho c}{2}\left(\frac{1}{n_{2}}+\frac{1}{\eta_{3}}\right),
\end{gathered}
$$

where the upper signs in equation (13) are associated with waves travelling in the direction of increasing $r$ and the lower signs are associated with waves travelling in the direction of decreasing $r$. The expression $P_{n}^{ \pm}$ is obtained from $P_{n}$ and equation (12), that is, when the plus sign is used with $S$ in the expression $P_{n}$ we call it $P_{n}^{+}$, and when the minus sign is used, $P_{n}^{-}$. Thus $P_{n}^{+}$is associated with outgoing waves and $P_{n}^{-}$ with incoming waves.

We consider the viscoelastic plates to be initially unstressed and at rest. For $t>0$, the opening is subjected to a suddenly rising uniform shearing traction so that $s\left(r_{0}, t\right)$ is known. Thus, from equation (6), we have for shearing tractions suddenly applied and thereafter steadily maintained, that

$$
s\left(r_{0}, t\right) \sim \sum_{n=0}^{\infty} A_{n}\left(r_{0}\right) F_{n}\left[t-S\left(r_{0}\right)\right]=s_{0} H(t),
$$

where $s_{0}$ is a constant measuring the strength of the source and $H(t)$ is the Heaviside unit function defined in (8). Thus, we choose [4], [17] 


$$
\begin{gathered}
A_{n}\left(r_{0}\right)=\left\{\begin{array}{lll}
\varepsilon_{0} & \text { if } n=0, \\
0 & \text { if } n>0 \text { or } n<0,
\end{array}\right. \\
S\left(r_{0}\right)=0, F_{0}=H(t) .
\end{gathered}
$$

The solution of equation (5), for outgoing waves generated by the above boundary stress, is given by

$$
\begin{aligned}
& s \sim \sum_{n=0}^{\infty} A_{n}(r)[t-S(r)]^{n_{H}}[t-S(r)] / n !, \\
& S=\int_{r_{0}}^{r} d \tau / c(\tau),
\end{aligned}
$$

where the $A_{n}$ are given recursively by (13) (with the upper signs) in conjunction with (15). The solution (16) applies up until the outgoing wave strikes either a boundary or an interface. We will show that reflected waves are produced at a boundary while both reflected and transmitted waves are produced at an interface between two media. The first-term approximation to our solution is

$$
s \sim s_{0}\left[\frac{p(r)}{p\left(r_{0}\right)}\right]^{\frac{1}{2}} \exp \left\{-\int_{r_{0}}^{r} \gamma(\alpha) d \alpha\right\} H(t-S),
$$

where $S$ is given in (16). This represents a transient stress wave which starts out from the opening at $r=r_{0}$ with amplitude $s_{0}$ and progresses in the direction of increasing $r$ with velocity $c(r)$. It is modulated by the factor $\left[p(r) / p\left(r_{0}\right)\right]^{\frac{1}{2}} \exp \left\{-\int_{r_{0}}^{r} \gamma(\alpha) d \alpha\right\}$. Further terms in the approximation may be obtained recursively from (13).

\section{The finite plate}

We consider the nonhomogeneous finite viscoelastic plate $r_{0} \leq r \leq r_{1},|z| \leq d / 2$, with a stress-free boundary at $r=r_{1}$, to have the surface of its cylindrical opening subjected to a suddenly rising uniform shearing traction of the type described in the preceding section. A transient stress is produced which leaves the boundary of the hole at 
time $t=0$ and travels with the velocity $c(r)$ in the direction of increasing $r$. This outgoing wave has been discussed in the preceding section and its asymptotic representation is given by (16) with the amplitude functions $A_{n}$ being given by (13) and (15), where the upper signs in (13) are chosen to correspond to an outgoing wave. From now on we will denote this outgoing wave by $s^{i}$ and its amplitude and phase functions by $A_{n}^{i}$ and $S^{i}$, respectively, implying that it is the wave which is incident at the stress-free boundary $r=r_{1}$.

We now assume that at the stress-free boundary there is produced a reflected wave $s^{r}$ which can be represented by

$$
s^{r} \sim \sum_{n=0}^{\infty} A_{n}^{r}(r) E_{n}\left[t-S^{r}(r)\right], A_{n}^{r} \equiv 0 \text { for } n<0 .
$$

The amplitude functions $A_{n}^{r}$ in (17) satisfy the transport equation (11). Its solution for an incoming wave which leaves $r=r_{1}$ and proceeds in the direction of decreasing $r$ is given by (13) with the lower signs, and $r_{1}$ replacing $r_{0}$. The phase function $S^{r}$ is given by equation (12) with the minus sign, and $r_{1}$ replacing $r_{0}$. We justify our assumption that a reflected wave is produced at $r=r_{1}$ by demonstrating that the boundary condition on $r_{1}$ can be satisfied by $s=s^{i}+s^{r}$. The boundary condition will also give the necessary initial conditions for the eikonal and transport equations thereby enabling us to formally determine the reflected wave.

Up until time $t=s^{i}\left(r_{1}\right)$ the solution is given by the incident wave $s^{i}$ which has been completely determined in the preceding section. At $t=S^{i}\left(r_{1}\right)$ the incident wave has arrived at the boundary $r=r_{1}$, and applying the boundary condition of vanishing stress we obtain 


$$
\begin{aligned}
& A_{n}^{r}\left(r_{1}\right)=-A_{n}^{i}\left(r_{1}\right), n=0,1,2, \ldots, \\
& S^{r}\left(r_{1}\right)=S^{i}\left(r_{1}\right) .
\end{aligned}
$$

Then, because $A_{n}^{i}\left(r_{1}\right)$ and $S^{i}\left(r_{1}\right)$ are known from the work of the preceding section, the reflected wave may be completely determined. It is given by (17) with the $A_{n}^{r}$ and $S^{r}$ being given by

$$
\begin{aligned}
& A_{n}^{r}(r)=-A_{n}^{r}\left(r_{1}\right)\left[p(r) / p\left(r_{1}\right)\right]^{\frac{3}{2}} \exp \left\{+\int_{r_{1}}^{r} \gamma(\alpha) d \alpha\right\} \\
& +\left(\frac{3}{2}\right) \int_{r_{1}}^{r} c(\tau)[p(r) / p(\tau)]^{\frac{1}{2}} \exp \left\{-\int_{r}^{\tau} \gamma(\alpha) d \alpha\right\} P_{n}^{-}(\tau) d \tau, n=0,1, \ldots,
\end{aligned}
$$

and

$$
S^{r}(\boldsymbol{r})=\left(\int_{r_{0}}^{r_{1}}-\int_{r_{1}}^{r}\right)(d \tau / c(\tau))
$$

The solution $\delta^{r}$ will then apply for $S^{i}\left(r_{1}\right)=S^{r}\left(r_{1}\right)<t<S^{r}\left(r_{0}\right)$. At time $t=S^{r}\left(r_{0}\right)$ the reflected wave $s^{r}$ arrives at $r=r_{0}$ and the boundary conditions there must be satisfied. The process is then repeated and in this way any number of reflections can be treated in a straightforward manner. The first-term approximation to the reflected wave is

$$
s^{r} \sim-s_{0}\left[p(r) / p\left(r_{0}\right)\right]^{\frac{z_{r}}{2}} \exp \left\{-\left(\int_{r_{0}}^{r_{1}}+\int_{r}^{r_{1}}\right) \gamma(\alpha) d \alpha\right\} H\left(t-S^{r}\right),
$$

where $S^{r}$ is given by equation (20). Further terms in this approximation may be obtained recursively from (19).

\section{The bi-viscoelastic infinite plate}

We consider the infinite nonhomogeneous plate $r_{0} \leq r<\infty$, $|z| \leq d / 2$, composed of two adjoining four-parameter viscoelastic media which we call medium " $a$ " and medium " $b$ ". The interface between these two media is at $r=\bar{r}$. We denote the wave speed and density of the two 
media by $c_{a}, c_{b}, \rho_{a}$, and $\rho_{b}$, and functions evaluated at the interface will be denoted by a bar, that is, $S^{i}(\bar{r})=\bar{s}^{i}$. The boundary conditions at the interface require that $\bar{s}_{a}=\bar{s}_{b}$ and $\bar{v}_{a}=\bar{v}_{b}$.

At the boundary of the cylindrical opening we apply the suddenly rising uniform shearing traction discussed in the preceding sections. A transient stress wave is produced which leaves the boundary of the opening at time $t=0$ and travels with the velocity $c_{a}(x)$ in the direction of increasing $r$ through medium $a$. This wave has been discussed in Section 3 and the solution given there will be valid up until such time as the outgoing wave meets the interface $r=\bar{r}$ between medium $a$ and medium $b$. We assume that when the incident wave strikes the interface $r=\bar{r}$, there is produced both a reflected wave which travels in the direction of decreasing $r$ into medium $a$, and a transmitted wave which proceeds from the interface into medium $b$. Due to the linearity of the equations, the stress field can be obtained by superposition. We assume that

$$
\begin{aligned}
& s_{a}=\sum_{n=0}^{\infty} A_{n}^{i} F_{n}\left(t-S^{i}\right)+\sum_{n=0}^{\infty} A_{n}^{r} F_{n}\left(t-S^{r}\right), \\
& s_{b}=\sum_{n=0}^{\infty} A_{n}^{t_{F}}\left(t-S^{t}\right), \\
& v_{a}=\sum_{n=0}^{\infty} B_{n}^{i_{F}}\left(t-S^{i}\right)+\sum_{n=0}^{\infty} B_{n}^{r_{F}}\left(t-S^{r}\right), \\
& v_{b}=\sum_{n=0}^{\infty} B_{n^{2}}^{t_{n}}\left(t-S^{t}\right),
\end{aligned}
$$

where the superscripts $i, r$, and $t$ refer to incident, reflected, and transmitted waves, respectively. The form for the velocity field in (2l) implies that the $A_{n}^{j}$ and $B_{n}^{j}$ are related by

$$
B_{n}^{j}=-(1 / \rho)\left[S^{j^{\prime}} A_{n}^{j}-A_{n-1}^{j^{\prime}}-(2 / r) A_{n-1}^{j}\right], n=0,1, \ldots, j=i, r, t,
$$

where $S^{j^{\prime}}=c^{-1}$ for the outgoing waves and $S^{j^{\prime}}=-c^{-1}$ for the incoming wave; equation (22) yields the well-known result from the conservation of 
momentum across a steadily moving discontinuity, that is, $A_{0}^{j}=\rho_{0}^{j},[4]$. Inserting (20), (21), and (22) in the boundary conditions $\bar{s}_{a}=\bar{s}_{b}$ and $\bar{v}_{a}=\bar{v}_{b}$, we find that

$$
\bar{s}^{i}=\bar{s}^{r}=\bar{s}^{t} \text {, }
$$

and

$$
\begin{aligned}
& \bar{A}_{n}^{r}-\bar{A}_{n}^{t}=-\bar{A}_{n}^{i}, \\
& \bar{A}_{n}^{r}+\overline{I A}_{n}^{t}=\bar{A}_{n}^{i}-\bar{f}_{n-1}^{i}-\bar{f}_{n-1}^{r}+\bar{I} \bar{f}_{n-1}^{t},
\end{aligned}
$$

where

$$
\bar{I}=\bar{\rho}_{a} \bar{c}_{a} / \bar{\rho}_{b} \bar{c}_{b}, \quad f_{n-1}^{j} \equiv c\left[A_{n-1}^{j^{\prime}}+\frac{2}{r} A_{n-1}^{j}\right], j=i, r, t .
$$

Solving the system given by equations (24) we have

$$
\begin{aligned}
& \bar{A}_{n}^{r}=\left[(1-\bar{I}) \bar{A}_{n}^{i}-\bar{f}_{n-1}^{i}-\bar{f}_{n-1}^{r}+\bar{I} \bar{f}_{n-I}^{t}\right](I+\bar{I})^{-1}, \\
& \bar{A}_{n}^{t}=\left[2 \bar{A}_{n}^{i}-\bar{f}_{n-1}^{i}-\bar{f}_{n-1}^{r}+\bar{I}_{n-1}^{t}\right](1+\bar{I})^{-1} .
\end{aligned}
$$

Thus, since $\bar{S}^{i}$ and $\bar{A}_{n}^{i}$ are known, the initial values at the interface are known, and the procedures of the preceding section may be applied to obtain the formal solution for the reflected and transmitted waves.

One interesting result from (26) is that, in the limiting case of $\bar{I}=1$ (that is, $\bar{\rho}_{a} \bar{c}_{a}=\bar{\rho}_{b} \bar{c}_{b}$ ), there is no reflected wave only if $\bar{f}_{n-1}^{i}=\bar{f}_{n-1}^{t}-\bar{f}_{n-1}^{r}$ for all $n \geq 1$. Thus, even though the impedance is continuous at $\bar{r}$ it is still possible that a wave will be reflected. Note that, in the case $\bar{I}=1$, the reflected wave has no discontinuity since $\bar{A}_{0}^{r}=0$. This was pointed out for the case of one-dimensional elastic waves by Cooper [4].

\section{Discussion}

We have presented a formal technique for obtaining asymptotic 
representations of the solution functions for radially symmetric transient stress waves in nonhomogeneous four-parameter viscoelastic media. Methods for treating reflection at a free surface and reflection and transmission at an interface between two media were also presented. The asymptotic method is direct and involves only ordinary differentiation, integration, and algebra. Because of its directness it is invariably true that it is much simpler than the procedure which involves first finding the exact solution, and then its asymptotic expansion. Also, our technique does not depend on separation of variables.

When uniform shearing tractions are suddenly applied to the boundary of a transverse cylindrical opening in a nonhomogeneous viscoelastic plate and thereafter steadily maintained, it is found that a cylindrical shear wave of stress discontinuity is produced. This wave propagates outwards from the opening with a variable velocity which depends only on the rigidity $\mu_{1}$. It is modulated by a factor which depends on the moduli of shear viscosity and the modulus of rigidity associated with the Maxwell part of our four-parameter element (that is, $\mu_{1}$ ). When this outgoing wave meets a stress-free boundary a reflected wave is produced which then travels in the opposite direction. If this outgoing wave meets an interface between two nonhomogeneous media, both a reflected and a transmitted wave are produced. The method of treating this interface may be used to treat multiple interfaces. One interesting result was that reflected waves may be generated at an interface between two different nonhomogeneous media even though the impedances at the interface are matched. In this case the reflected wave does not possess a discontinuity.

\section{References}

[1] D.R. Bland, The theory of Zinear viscoelasticity (Pergamon Press, Oxford, London, New York, Paris, 1960).

[2] George B. Clark and Gerald B. Rupert, "Plane and spherical waves in a Voigt medium", J. Geophys. Res. 71 (1966), 2047-2053. 
[3] George B. Clark, Gerald B. Rupert and James E. Jamison, "Series transform solutions for Voigt transients", Quart. App 2. Math. 25 (1967), 349-361 (1968).

[4] Henry F. Cooper, Jr, "Propagation of one-dimensional waves in inhomogeneous elastic media", SIAM Rev. 9 (1967), 671-679.

[5] Henry F. Cooper, Jr and Edward L. Reiss, "Propagation and reflection of viscoelastic waves", J. Acoust. Soc. Amer. 38 (1965), 24-34.

[6] F.G. Friedlander, "Simple progressive solutions of the wave equation", Proc. Combridge Philos. Soc. 43 (1947), 360-373.

[7] R.D. Glauz and E.H. Lee, "Transient wave analysis in a linear timedependent material", J. App Z. Phys. 25 (1954), 947-953.

[8] Frank C. Karal, Jr and Joseph B. Keller, "Elastic wave propagation in homogeneous and inhomogeneous media", J. Acoust. Soc. Amer. 31 (1959), 694-705.

[9] J.B. Keller, R.M. Lewis and B.D. Seckier, "Asymptotic solution of some diffraction problems", Comm. Pure Appl. Math. 9 (1956), $207-265$.

[10] Morris Kline, "An asymptotic solution of Maxwell's equations", Corm. Pure App I. Math. 4 (1951), 225-262.

[11] H. Kolsky, Stress waves in solids (Dover, New York, 1963).

[12] H.A. Lang, "Two verifications of the Karal-Keller theory of wave propagation", J. Acoust. Soc. Amer. 34 (1962), 785-788.

[13] T.M. Lee, "Spherical waves in viscoelastic media", J. Acoust. Soc. Amer. 36 (1964), 2402-2407.

[14] Robert M. Lewis and Joseph B. Keller, Asymptotic methods for partial differential equations; the reduced wave equation and Maxwell's equations (Report EM-194, Courant Institute of Mathematical Sciences, New York University, New York, 1964).

[15] Jacob Lubliner, "Cylindrical wave in a viscoelastic solid", $J$. Acoust. Soc. Amer. 34 (1962), 1706-1710.

[16] R.K. Luneburg, Mathematical theory of optics (University of California Press, Berkeley, Los Angeles, 1964). 
[17] T. Bryant Moodie, "On the propagation of radially symmetric waves in nonhomogeneous isotropic elastic media", Utilitas Math. 2 (1972), 181-203.

[18] Im K. Park, Edward L. Reiss, "Oscillatory impact of an inhomogeneous viscoelastic rod", J. Acoust. Soc. Amer. 47 (1970), 870-874.

[19] Gerald Bruce Rupert, "A study of plane and spherical compressional waves in a Voigt viscoelastic medium", ( $P h D$ thesis, University of Missouri at Rolla, 1964).

Department of Mathematics,

University of Dundee,

Dundee,

Scotland. 